\title{
Patient and Carer Experience of Nutrition Care Throughout and Beyond Treatment for Head and Neck Cancer: a Qualitative Longitudinal Study
}

Joanne Hiatt ( $\sim$ Joanne.Hiatt@health.qld.gov.au )

Royal Brisbane and Women's Hospital https://orcid.org/0000-0001-7019-6545

\section{Adrienne Young}

Royal Brisbane and Women's Hospital

\section{Teresa Brown}

Royal Brisbane and Women's Hospital

\section{Merrilyn Banks}

Royal Brisbane and Women's Hospital

Judy Bauer

University of Queensland - Saint Lucia Campus: The University of Queensland

\section{Research Article}

Keywords: qualitative research, nutrition care, head and neck cancer, patient and carer experience

Posted Date: June 18th, 2021

DOI: https://doi.org/10.21203/rs.3.rs-556570/v1

License: (c) (1) This work is licensed under a Creative Commons Attribution 4.0 International License. Read Full License

Version of Record: A version of this preprint was published at Supportive Care in Cancer on August 14th, 2021. See the published version at https://doi.org/10.1007/s00520-021-06484-3. 


\section{Abstract}

Nutrition care plays a critical role in optimising outcomes for patients receiving treatment for head and neck cancer (HNC), with carers playing an important role in supporting patients to maintain nutrition intake. This study explores patient and carer experience of nutrition care from diagnosis of HNC to oneyear post treatment completion to identify areas for improvement of service delivery. A longitudinal qualitative study design was used with a heterogeneous sample of 20 patients and 15 carers of patients undergoing curative intent treatment for HNC. Interviews conducted at four time points provided a total of 117 interview datasets that were analysed using reflexive thematic analysis based on Gadamerian hermeneutic inquiry. Patient and carer experiences were reflected in two primary themes; 1 ) the battle to maintain control, and 2) navigating the road ahead. This research identifies the need to co-design strategies to improve nutrition care that is inclusive of patients and carers.

\section{Background}

Head and neck cancer (HNC) represents $5 \%$ of all cancers and cancer deaths worldwide [1]. Alcohol and tobacco use are established risk factors for HNC [2], with human papillomavirus (HPV) infection a cause of increasing incidence in younger adults [3]. The main treatment methods for HNC are surgery, radiotherapy and chemotherapy, with many patients undergoing multimodal treatment [4]. Patients with HNC may experience difficulty with nutrition intake prior to treatment due to the psychological impact of their diagnosis, tumour location and lifestyle factors $[5,6]$. Treatment toxicities often cause severe physical and psychosocial side effects further impacting nutrition intake [7]. Consequently, many patients with $\mathrm{HNC}$ are at high risk of malnutrition prior to, throughout and beyond their treatment period.

Despite the provision of evidence-based nutrition support, malnutrition rates remain high, increasing patient risk of unplanned hospital admissions and mortality, and decreasing quality of life and treatment $[8,9]$. While a multidisciplinary approach is critical in the provision of best practice nutrition care to patients with HNC, studies have highlighted the importance of the carer in the provision of nutrition care [10]. Exploring patient and carer experiences may provide greater insight into ways to optimise nutrition care. A systematic review of qualitative studies investigating patient and carer experiences highlighted the paucity of data exploring the carer experience of nutrition care and the need for further research exploring patient and carer experience of nutrition care across different treatment modalities, before, during and beyond the treatment period [11].

The aim of this study was to explore and understand patient and carer experiences of nutrition care from diagnosis of $\mathrm{HNC}$ to one-year post treatment completion, with the purpose of identifying areas for improving service delivery.

\section{Materials And Methods}

\section{Study design}


This study focused on nutrition care with preunderstanding rooted in our previously published systematic review [11]. A prospective longitudinal study was undertaken by conducting interviews with patients and carers of patients with HNC prior to treatment commencing, and two weeks, three months and 12 months post treatment completion. Interviews and analysis were undertaken by the principal investigator $(\mathrm{JH})$, a dietitian working in $\mathrm{HNC}$ but not providing direct care of participants. A narrative interview method was used to explore patient and carer experiences of nutrition care [12]. Reflexive thematic analysis based on Gadamerian hermeneutic inquiry was used in the steps of data collection, interpretation and analysis [1315]. This provided the opportunity for the principal investigator to acknowledge their personal experiences working in $\mathrm{HNC}$ when analysing and interpreting the text.

\section{Setting}

In current practice, patients diagnosed with HNC attend a multidisciplinary HNC clinic where they are screened for malnutrition by a dietitian. High risk patients are referred for dietetic intervention and prophylactic gastrostomy placement prior to treatment aligning with local hospital protocol [16]. As per evidence-based guidelines, patients undergoing radiotherapy are referred for weekly appointments with the dietitian and ongoing follow up fortnightly for a minimum of 6-weeks post treatment completion [17]. Patients undergoing surgery requiring enteral feeding are seen by the ward dietitian to review commencement of enteral nutrition support and transition to oral intake with follow up appointments scheduled fortnightly as needed. Follow up care for patients is dependent on their local health service district location.

\section{Participants}

Patients and carers were recruited from a multidisciplinary HNC clinic at a tertiary/quaternary hospital in Brisbane, Australia. Patients and carers were approached to participate if they could speak English, aged over 18 years, and presenting with their first diagnosis of $\mathrm{HNC}$ and suitable for curative intent treatment. Purposive sampling was used to select patients and carers from a range of treatment modalities, tumour size, geographical location, gender and age.

\section{Consent}

Participants were provided with verbal and written information on the study on presentation to the multidisciplinary HNC clinic at the time of their diagnosis. Consent to participate was obtained one week later.

\section{Procedures}

Interviews were completed by the principal investigator between June 2018 and April 2020 and were conducted prospectively over the first year from diagnosis. Each interview began with a broad definition of nutrition care, and participants were asked to describe their experiences in accessing support with their nutrition intake at each separate time point. Themes emerging from earlier interviews were incorporated into the interview guide in subsequent interviews. Interviews were held face-to-face or via telephone as per 
participant preference. Interviews lasted between 10 and 60 minutes and were audio recorded and transcribed verbatim by the principal investigator.

\section{Data analysis}

The understanding of the interview text was carried out through a back and forth process between the parts and the whole as outlined in the six-phase process for thematic analysis by Braun and Clarke [13]. The 'text' was fused with the experiences of the principal investigator to facilitate interpretation and a complete understanding of the data set. To support a reflexive approach, weekly meetings were conducted with the second researcher (AY; dietitian not working in HNC), to discuss individual influences on the process and outcomes. This was used to identify how the clinical experience of the principal investigator may enhance interpretation of the data. The Consolidated Criteria for Reporting Qualitative Research (COREQ) checklist was used to ensure comprehensive reporting [18].

\section{Ethics approval}

Ethics approval was obtained by the Royal Brisbane and Women's Hospital Research \& Ethics Committee, Brisbane and the Ethics Committee of the University of Queensland, Brisbane, Australia (HREC/17/QRBW/681), with Site Specific Approval for the study to be conducted at the Royal Brisbane and Women's Hospital.

\section{Results}

\section{Participant demographics}

Thirty-five participants (20 patients and 15 carers) were enrolled in the study. Diagram 1 outlines participant recruitment and retention in the study, with demographics outlined in Table 1. There was a relatively even spread of patient participants across different treatment modalities. However, there were fewer carer participants of patients undergoing surgery alone. Three patient-carer dyads participated in the study. Eleven patient participants and 12 patients being cared for by carer participants had a feeding tube placed over the duration of their care.

\section{Table 1: Participant demographics}




\begin{tabular}{|c|c|c|}
\hline & Patient $(n=20)$ & Carer $(n=15)$ \\
\hline \multicolumn{3}{|l|}{ Gender } \\
\hline Male & 15 & 2 \\
\hline Female & 5 & 13 \\
\hline \multicolumn{3}{|l|}{ Age category } \\
\hline $20-29$ & 1 & 0 \\
\hline $30-39$ & 0 & 1 \\
\hline $40-49$ & 4 & 3 \\
\hline $50-59$ & 5 & 5 \\
\hline $60-69$ & 6 & 5 \\
\hline $70-79$ & 4 & 1 \\
\hline \multicolumn{3}{|c|}{ Supported by (patient) or providing support for (carer) } \\
\hline Spouse/family & 15 & 15 \\
\hline Friend & 1 & 0 \\
\hline Alone (patient) & 4 & $\mathrm{n} / \mathrm{a}$ \\
\hline \multicolumn{3}{|c|}{ Tumour location (patient) } \\
\hline Oral cavity & 10 & 7 \\
\hline Oropharynx & 7 & 5 \\
\hline Nasopharynx & 1 & 2 \\
\hline Hypopharynx & 1 & 0 \\
\hline Larynx & 1 & 1 \\
\hline \multicolumn{3}{|c|}{ Treatment modality (patient) } \\
\hline Surgery & 6 & 3 \\
\hline Surgery + PORT & 6 & 7 \\
\hline Chemoradiotherapy & 8 & 5 \\
\hline \multicolumn{3}{|l|}{ T stage (patient) } \\
\hline T0-2 & 10 & 8 \\
\hline \multicolumn{3}{|c|}{ HPV: Human papillomavirus } \\
\hline PORT: Post-operative & apy & \\
\hline
\end{tabular}




\begin{tabular}{|lll|}
\hline & Patient $(\mathbf{n = 2 0})$ & Carer $(\mathbf{n}=\mathbf{1 5})$ \\
\hline T3-4 & 10 & 7 \\
\hline HPV p16 status (patient) & & \\
\hline Positive & 5 & 12 \\
\hline Negative/not stated & 15 & \\
\hline Nutrition support (patient) & & 3 \\
\hline Oral & 4 & 6 \\
\hline Gastrostomy & 4 & 3 \\
\hline Nasogastric tube & 8 & 3 \\
\hline Not applicable & 4 & \\
\hline Geographical location & & 9 \\
\hline Urban & 12 & 5 \\
\hline Regional & 4 & 1 \\
\hline Rural & 4 & \\
\hline HPV: Human papillomavirus & & \\
\hline PORT: Post-operative radiotherapy & \\
\hline
\end{tabular}

Two main themes were identified. These included: (1) the battle to maintain control; and (2) navigating the road ahead. Within each theme, subthemes were identified as outlined in Tables 2 and 3. 
Table 2

Quotes related to theme 1: the battle to maintain control

\section{Reference Quote number}

Subtheme 1.1: Health and nutrition beliefs

1 "... a friend of ours she actually had cancer and she beat it not by undergoing any therapy, but she just watched what she ate. So, everything was pretty much all natural and she beat the cancer that way" (Carer 2, baseline).

$2 \quad$ "Yeah I kept asking the dietitian at the hospital what shouldn't I be eating, and they kept saying oh no you can eat anything I just don't agree with that, so I think I'll keep doing what I'm doing" (Patient 7, baseline).

"Absolutely the support has been amazing. Amazing. We have had the most amazing support. The support all the Dietitians and the speech therapy and the physio, everyone has been there and the psychologist, everyone, the treatment has been fantastic, and the information given and the recipes, you know like scrambled eggs and lots of different little recipes to try to help. We got a lot of information" (Carer 13, 2 weeks).

"I've tried to be more like cautious and healthy, in terms of not eating out a lot, or like not eating processed food, everything that is considered maybe like it may cause or like increase risk of getting cancer, so..." (Patient 2, 3 months).

"... one of the interesting things that I found was the at times mismatch between what is proven clinical science and what is emerging possibly better ways to look at cancer in terms of what you put into your body so I'm talking health supplements, whether its vitamins or the like and that's an interesting thing to talk to people about. I fully understand the way the medical profession approaches it and goes with the proven science when you're dealing with life and death, um how do you get around particularly with what you do how do you get around with what you've got is probably accurate emerging science but it's still emerging and it's not yet clinically accepted" (Patient 5, 12 months).

Subtheme 1.2: Weight loss rollercoaster

6

"Look I mean it's almost inevitable anyway I mean I'm losing weight now so why not celebrate" (Patient 14, baseline).

7

"I didn't expect the weight loss to be as drastic as it was, and um, I didn't expect the mental battle... and I've got this constant battle about 'I've gotta get through this, I've gotta get through this" (Patient 7, 2 weeks).

"As the degree of weight loss stacked up after that it started to worry me a little bit and I realised that as well as shedding a bit of unnecessary fat I also shed muscle mass. That surprised me and I've to make a point of restoring that muscle mass because I like to have it" (Patient 5, 2 weeks).

I found it very confronting that he was losing all this weight and I felt helpless I couldn't seem to be able to do anything to assist him (Carer 13, 12 months).

"He is happy with the weight he's lost about 7-8kg which he needed to in the beginning so... He's trying to stay that weight (Carer 4, 2 weeks). ok with that" (Patient 5, 12 months). 


\section{Reference Quote number}

Subtheme 1.3: Acceptance of feeding tube

12

"So, she said do what you have to do but because I'm having the radiation, I may have problems swallowing and eating and drinking so we're going to have a PEG put in. Which means then I can monitor exactly what's going in" (Patient 11, baseline).

13

"I was likely to get to a stage where I would have to be put on a tube to feed. I actively avoided that which I'm really glad I managed to achieve. I think my expectations managed my knowledge at the time" (Patient 5, 2 weeks).

14 "yeah, I don't think it's right, I don't think it should have been a choice. I think he should have said this is the way we're going, and you've got to opt out" (Patient 7, 2 weeks).

15

"No, no, like after the surgery I was complaining about wanting to get the tube out of my nose and throat and they kept explaining to me why it had to be in and why I couldn't swallow straight away but of course I had to complain about it" (Patient 13, 2 weeks).

16

"But he needed it [PEG], like he wouldn't be where he was or where he is now if he hadn't of had it now. It's necessary. I just think it's horrible, bad, not nice. I wouldn't think it would be a nice process to have to go through and have to deal with. No" (Carer 6, 12 months).

Subtheme 1.4: Gatekeeper role of patient

"But there are a lot of loved ones around me and it was just too much in the end to see them every day or answer a phone call every 5 minutes. People are genuinely concerned but that's why I didn't bring any support people here with me. It's easier to just get over this first hurdle on my own to give me a chance to absorb everything that's thrown in front of me and to get into a pattern or routine, a personal routine. Once I bounce off the ropes of the hospital here hopefully, I can be a source of courage in their lives, but I didn't want anybody to be upset or concerned or worried. I got myself into this predicament, so I need to learn how to struggle through it, but I don't know that it's going to be a struggle" (Patient 9, baseline).

18

"Nope. I mean the issue is that everyone talks to [patient name], but [patient name] doesn't cook the meals. I think the first question they need to ask patients is 'do you live on your own' and 'do you cook your own meals' that should be their first question. If the patient says no, I don't cook a lot of meals my wife does then you should go good let's go talk to the wife. I hope that gives you an impression of my perception so far..." (Carer 14, 2 weeks).

19

"... we went back to see the dietitians at Hervey bay hospital they actually suggested that maybe they give us extra protein for him um but he declined it wanted to do it all on his own so the support was definitely there but obviously the patient wasn't that willing at the time but he wasn't wanting to stop using his throat and he wanted to do it alone but I found that very hard. But well basically I had to do little samples, he just had to have say 6 meals a day, very small meals a day um but yeah finding, finding food the good food for him all the time I found difficult yep" (Carer 7, 3 months).

Subtheme 1.5: Invisible load of carer 


\section{Reference Quote \\ number}

20

"I'm trying to eat what I can as best I can because if I get run down that's the biggest problem you know so I was starting to get a bit the other week but l've been getting run down because I've had to be down here every week for this eye operation so I'm up here again for this one so hopefully after this it will be fortnightly or monthly I'm hoping anyway just to slow down a bit I think that's why I was getting knotted up to because I was running around and going back and just living off whatever that sort of thing" (Carer 1, 12 months).

"Yeah and I'm sure there are a lot of carers that end up burnt out and I don't get anything from the government because I made too much money in the past. There is very little for carers these days anyway so you know I think yeah it's a bit disappointing really, the whole thing but yeah I definitely feel I wasn't supported when he was going through the treatment and that is partially why I was so burnt out and even walking back into the hospital I get pretty severe anxiety and I'm not the one getting treated" (Carer 10, 12 months). 
Table 3

Quotes related to theme 2: navigating the road ahead

\section{Reference Quote number}

Subtheme 2.1: Challenges comprehending information provided

"To be honest all the information was there and like everyone sort of said this and that would happen but yeah I don't think you could ever prepare for it like who in the world can prepare for the unknown um I mean yeah if I'd had to do it again you'd think bloody twice but um knowing yeah and as you could probably agree, everyone's body reacts differently I mean I saw other people in worse situations in me and other people I was in worse situation than them and I guess it just is what it is" (Patient 11, 12 months).

Subtheme 2.2: Information accessibility and inclusivity

"Um really we weren't told what he could and couldn't eat so we just had to work out what he felt comfortable eating and because it was his mouth and his throat his food options were pretty limited so with the reconstruction of his lip he had to work out what he could get over his lip so um he couldn't suck on a straw because he couldn't purse his mouth like that so everything had to be cut really small. As far as assistance from the hospital it was a disaster, we didn't really get any" (Carer 12, 2 weeks).

"...there's a lot of information out there, um some of it, maybe too much information sometimes um some contradict each other as well so you've really got to find your way through it" (Carer 4, 3 months).

If there was a place where we would see we could learn we could talk to other patients, you know a place like that where its practical and hands on. You know if there was something like that here it would take all the guessing away of what we need to be doing that way we can go home and do exactly what we need because we would have an organised dietary plan, a menu for the week of an idea of where to go on the bloody internet to find what we need. It would sure beat ringing up the cancer care lady and asking her about my dietary requirements, you know I don't think that's very fair to other people that need her time. You know if there was a place we could go with a kitchen where other people can go also and we can ask questions you know just to give us a chance to know what we should be doing because a lot of it is just guessing and taking it day by day (Patient 9, 3 months).

Subtheme 2.3: Moving away from the hospital environment

"All the hospital staff in their different areas of profession their support has been really wonderful. I just need to soak all that up and understand where everybody fits so I know where I can draw on those resources and support and when I need it. I just have to get into a pattern, and I'd like to have the solidarity in myself to process it" (Patient 9, baseline).

"but the other people like the Aboriginal people they don't know, they don't care I don't know if they think it's contagious and they might catch it. I've got so many questions to ask but there's no appointments no follow up. Some days I want to get out there and bang my head against their door and let them know we're normal people, we need assistance and sometimes we just need someone who we can turn to or have a yarn to or a cup of tea with" (Patient 9, 2 weeks).

"So, I've come a long way yeah, it's good. I turned the corner and pushing forward. On Friday get the PEG out and I'm hoping to go back to work next week or the week after yeah, the boss is happy if I want to do 1 hour, 4 hours, 5 hours whatever I can handle" (Patient 12, 3 months). 


\section{Reference Quote number}

\section{8}

"yeah most definitely you know I'm not saying like we still had that support here but once I came home yeah I felt um everything felt so much, I just felt it was a bit tougher for me to keep his nutrition up once he started cutting back on the Fortisip and they still weighed him and all that and you see it's all up to the patient and that's probably why the carer is so important because the patient sometimes doesn't want to keep going with the tube and um yeah so I had to sort of I had to be bit tough there with [patient name]to keep his nutrition up and he did lose a lot of muscle mass I did find that bit a bit tough yeah" (Carer 7, 3 months).

"Having those things to look forward to is huge like l've always been one to have something to look forward to I think it helps me and [patient namelyou know we just say will we go for a drive today and we just go for a drive, its flipped us its completely flipped out lifestyle, we look like we live in a tip some days but you know what you've got to get out and make some memories and that's what we do now and things that used to worry us don't worry us much anymore" (Carer 4, 12 months).

Subtheme 2.4: Peer support

"Oh, prepared only by reading things and I didn't have a book but [radiation oncologist] gave us a book the swallows I think it was called I read, [patient name]didn't read them he didn't want to read them but I read them I got a lot out of that. It's useful to read other people's experiences because every person has a totally different experience just being down there for 8 weeks and talking to people in the waiting room every single one of them even though it was similar treatment all reacted differently um so yeah it was really educational the whole thing um even though you're getting told a lot of things it's all a first" (Carer 7, 2 weeks).

"It's not a formal thing it's just something we do, we do it on the last Tuesday of the month at the the town hall and we just get together, the carers, talking about things that things we had difficulty with, just sharing, it's just sharing information and different ways to tackle things. We have all gone through a different hell of some description with their radiation, with their journey. It's been very good actually but there's nothing really formal with it but it's a support group for everybody. It's very good. It's really worth while and I said to one of the radiation doctors the other day, [radiation oncologist]and I said it's a very worthwhile place for your patients going through all these problems (Carer 13, 12 months).

12

"Um I haven't accessed external support other than for my own mental health um and I'm aware that I could go to cancer council and go to groups and things like that, but [patient name]said he didn't want to do that" (Carer 14, 12 months).

\section{Theme 1: The battle to maintain control}

\section{Subtheme 1.1 Health and nutrition beliefs}

At each interview timepoint, patients and carers described having a focus on healthy eating. For some this translated to eliminating foods they believed to be linked to cancer and disease, particularly when they had heard stories from others claiming to have beaten cancer through diet alone (Table 2, quote 1). 
Some participants described a lack of acknowledgement of their own views on nutrition and health by the dietitian. Consequently, some patients withdrew from nutrition care provided to them throughout treatment when nutrition recommendations provided did not align with their nutrition beliefs (Table 2 , quote 2).

Despite participants expressing strong views on nutrition and health, many highly valued and accepted nutrition support provided to them throughout their treatment from the dietitian and multidisciplinary team (Table 2, quote 3 ). At the end of the treatment period, the overall sense was that many patients and carers wanted to step away from high protein, high energy food and fluid recommendations, as their focus shifted to making lifestyle and dietary changes to prevent cancer recurrence (Table 2, quote 4).

One-year post treatment, some patients reflected on wanting more open discussions with healthcare professionals about the use of vitamin and mineral supplementation and complementary alternative medicine to support them through their treatment and recovery (Table 2, quote 5).

\section{Subtheme 1.2: Weight loss rollercoaster}

Prior to treatment, patients and carers expressed their views on weight loss as a positive outcome. (Table 2 , quote 6 ). As the acuity of treatment side effects worsened, impacting on nutrition intake, weight loss for many became uncontrolled. This had a negative effect on the psychological wellbeing of many patients and carers as it also included a loss of strength and muscle mass (Table 2, quote 7,8 ).

Some carers feared that weight loss was a sign that the patient was not getting better, while others felt helpless despite their efforts in providing care (Table 2, quote 9).

As weight loss became controlled again after treatment completion, patients and carers reflected positively on the experience, as it aligned with their goals of leading a healthy lifestyle. For many this included wanting to maintain a lower body weight while restoring muscle mass lost through the treatment period (Table 2, quote 10,11).

\section{Subtheme 1.3: Acceptance of feeding tube}

Patients undergoing chemoradiotherapy were accepting of recommendations to have a prophylactic gastrostomy tube placed when it was presented as a necessary part of treatment (Table 2, quote 12). Some patients made the decision to decline gastrostomy placement and described how this provided them with the motivation to maintain oral intake to avoid nasogastric tube placement (Table 2, quote 13).

One patient expressed resentment after having a nasogastric tube placed after declining prophylactic gastrostomy tube placement when it was presented as an optional part of treatment (Table 2, quote 14).

Despite being described as an unpleasant experience, surgical patients were accepting of nasogastric tube placement in theatre as they understood it was the only way they could be provided nutrition support 
when they were required to remain nil by mouth as they recovered from surgery (Table 2, quote 15).

Many carers highly valued the gastrostomy tube and viewed it as a necessary part of treatment, as it provided a solution to support the patient with nutrition intake when eating and drinking became too difficult (Table 2, quote 16).

\section{Subtheme 1.4: Gatekeeper role of the patient}

Some patients limited the amount of information they shared and support they accepted from friends and family in an effort to avoid being a burden to them. One patient described how the enormity of his diagnosis and treatment plan had left seeking solidarity to process the information himself first before accepting the support of loved ones (Table 2, quote 17).

While this was done by patients in a way to "protect" those trying to provide care, many carers expressed their frustration in being excluded from the provision of care. One carer described how nutrition care provided by healthcare professionals was not passed onto her by her partner and therefore not of value to the patient when the information provided did not take into account the practical aspects of nutrition support, including acknowledgement of the fact that she was the one responsible for grocery shopping and meal preparation (Table 2, quote 18).

After the end of the treatment period, some carers found it difficult when they still wanted to maintain contact with healthcare professionals for nutrition support but felt this was not an option available to them if the patient was not accepting of follow up care (Table 2, quote 19).

\section{Subtheme 1.5: Invisible load of carer}

Prior to treatment commencing, carers described the importance of maintaining their strength and having a positive outlook to support the patient. However, after supporting the patient through the treatment and recovery period, the psychological impact of taking on this role was emphasised. Carers described the stress they experienced in trying to maintain their physical health to ensure they had enough strength to continue to support the patient (Table 2, quote 20).

The biggest challenge carers described was the lack of support available to them in their carer role from healthcare professionals, and the negative impact this had on their psychological wellbeing. One carer described the severity of the psychological impact of providing care for her father without having any support for herself and consequently found it difficult to return to the hospital with her father for his follow up appointments (Table 2, quote 21)

\section{Theme 2: Navigating the road ahead}

\section{Subtheme 2.1: Challenges comprehending information provided}

Patients described feeling unprepared for the impact that treatment side effects had on their ability to eat and drink despite being provided with nutrition care from healthcare professionals. One year after 
treatment completion, patients reflected on how difficult it was to absorb information provided to them due to the enormity of their diagnosis and treatment. Some felt no amount of information would have helped them prepare for what lay ahead of them (Table 3, quote 1).

\section{Subtheme 2.2: Information accessibility and inclusivity}

Patients and carers of patients having radiation treatment felt well supported by healthcare professionals with nutrition care, as regular appointments with the dietitian were scheduled over their treatment period. In contrast, carers of surgical patients found it challenging to access nutrition support from healthcare professionals on the ward (Table 3, quote 2).

Some patients and carers looked up nutrition information and support on the internet. Carers valued the ease of access to information that the internet provided, however many struggled to filter out appropriate from non-appropriate sources of nutrition support, especially without the guidance of healthcare professionals (Table 3, quote 3 ).

Written information that was easy to understand was valued by patients and carers as they could refer back to it at any time. However, many described a desire to have more visual aids to refer to and to be provided with hands on practical sessions to increase self-efficacy to support nutrition intake. One patient felt that practical sessions including cooking classes would give them the confidence to action nutrition recommendations provided to them (Table 3, quote 4).

\section{Subtheme 2.3: Moving away from the hospital environment}

Prior to treatment commencing, many patients and carers described how much they valued having access to a multidisciplinary team of healthcare professionals to support them through their HNC journey (Table 3, quote 5).

Having access to a multidisciplinary team of healthcare professionals working in the same environment provided patients and carers with a feeling of security around their overall care. Consequently, some patients and carers from regional and rural locations found it difficult when the same level of support was not accessible or available to them when they returned home (Table 3, quote 6).

Patients remaining in the hospital district location who still had access to support from the multidisciplinary team after treatment completion, described a degree of self-disengagement from the hospital environment and healthcare professionals. Patients were happy to prioritise hospital appointments with surgeons or oncologists but otherwise felt their nutrition care was something that could be managed independently at home. Some patients described their focus now was on returning to work and 'getting back to normal' (Table 3, quote 7).

Many carers found it challenging where they no longer had frequent contact and support from healthcare professionals after treatment completion (Table 3, quote 8). However, as the acuity of the side effects of 
treatment eased, many carers felt some reprieve from the intensity of their caring role and described an appreciation of being back in their home environment (Table 3, quote 9).

\section{Subtheme 2.4: Peer support}

Patients and carers valued having access to information and support from other patients and carers that had been through treatment for HNC. This included being able to read their experiences either in the form of booklets, magazines or blogs online (Table 3, quote 10).

Others found it useful be able to talk to patients and carers they met throughout treatment or support groups as it provided the opportunity to talk through different strategies to support nutrition intake (Table 3 , quote 11).

Some carers felt that attendance at support groups was dependent on the patient's willingness to engage, rather than a support option for themselves (Table 3, quote 12).

\section{Discussion}

Our findings reveal two main themes describing patient and carer experience of nutrition care throughout and beyond treatment for HNC. While current evidence-based guidelines support appropriate and quality nutrition care for patients with HNC [17], findings from this study highlight tensions in the care relationships between patients, carers and healthcare professionals, as well as the changing nutritional needs and focus throughout the treatment and recovery journey.

'The battle to maintain control' describes the struggle many patients and carers experience in trying to regain a level of control in their life while juggling the psychological impact of their diagnosis and treatment [19]. Dietary changes and use of complementary alternative medicine (CAM), have been previously described by patients as a proactive step towards health improvement [20,21]. We found that patients and carers had difficulty engaging with healthcare professionals when they felt their views on nutrition and health were not acknowledged. Sources of dietary and CAM information can be misleading when they are more commonly provided by family and friends, health food stores and social media platforms [22]. As a step towards preventing disengagement and ensuring patient safety, this study highlights the need for healthcare professionals to provide patients and carers with the opportunity to discuss their nutrition views and beliefs and explore individual barriers and enablers to nutrition recommendations provided.

Weight loss in patients diagnosed with cancer can be distressing for patients and carers, having a negative impact on quality of life and functional status of patients $[11,23]$. While we found high levels of patient and carer distress when weight loss was uncontrolled, overall weight loss was viewed as a positive outcome. Negative emotion can be experienced when there is feeling of threat to wellbeing [24]. In our study, patients and carers described negative emotion two weeks after treatment completion when weight loss had escalated with the increasing acuity of treatment side effects. This demonstrates the need to address the psychological impact of weight loss on the patient and carer to alleviate feelings of 
distress, especially when carers may take this on as their responsibility. Levels of distress were further exacerbated with loss of muscle mass and strength. Approximately $70 \%$ of weight loss in patients with $\mathrm{HNC}$ is attributable to muscle loss, resulting in reduced strength and performance and increased risk of recurrence and mortality $[25,26]$. However, many patients and carers felt that weight loss aligned with their goals to lead a healthier future. This suggests a need for discussion with patients and carers around the impact of skeletal muscle depletion on survival. Recent studies have highlighted the value that body composition and muscle status evaluation measures hold in becoming part of routine clinical practice to facilitate early identification of sarcopenia $[27,28]$.

The literature on patient and carer experiences of enteral feeding highlight the stress and anxiety experienced in making a decision about tube placement [29]. This includes the role healthcare professionals play in influencing their decision, with many basing recommendations on personal experiences and perceptions [30,31]. Findings from our study align with recommendations that healthcare professionals should be provided with education and training about the different types of enteral feeding to ensure patients and carers are able to make a well-informed decision [30, 32]. Studies have found that patients with prior experience with enteral feeding are happy to support other patients in the decision-making process $[33,34]$. This presents an opportunity for peer support and learning through the experience of others when making key decisions about tube placement.

Some patients distance themselves from support provided to them from healthcare professionals, as well as family and friends [19]. We found that patient disengagement from care and support can create emotional distress for the carer, especially where they feel their own access to information and support from healthcare professionals is dependent on patient engagement. As previously described, while this study is focused on nutrition care, this also extends to their overall care, including accessing other sources of support from the multidisciplinary team [28]. Where carers play a key role in seeking out information to support the patient, it is important that healthcare professionals identify ways to overcome these barriers to ensure patients and carers have access to information and support [28].

'Navigating the road ahead' describes the effort made by patients and carers in looking forward to the future. Many patients and carers value having information they could refer to in order to support their nutrition intake. However, studies have shown that health literacy levels impact the understanding and interpretation of nutrition related information [35]. In this study, some patients expressed frustration in being provided with information when it did not translate to them having the confidence and skills to act on recommendations provided. A culinary intervention program developed for patients with cancer related fatigue found that access to experiential learning in the form of face to face and online cooking classes improved participants cooking skills, providing them with greater confidence to achieve their nutritional intake [36]. Furthermore, the use of visual aids including illustrations and videos can help patients have a greater understanding of information provided [37].

Over the years many patients and carers have increasingly turned to the internet including social media platforms to document their cancer journey, seek social support and obtain information [38]. The internet 
provides the opportunity for patients and carers to seek information and support regardless of geographical location and for carers to seek support independently of the patient. While this can be beneficial, there is the potential for harm, conflict and exploitation [39]. Many patients and carers in our current study sought out information and support online independently of advice or guidance from healthcare professionals. This highlights the need for healthcare professionals to guide patients and carers through available online resources to support evidence-based nutrition care.

For patients undergoing curative intent cancer treatment, there is an expectation to 'return to normality' at the end of the treatment period [40]. Studies have shown that normality can have a range of meanings from returning to life before diagnosis to creating lifestyle changes [19]. Many patients in this study looked forward to returning to work. While returning to work is viewed as a positive sign of moving on with life, it can also cause anxiety where patients still face difficulties eating and drinking, physical changes and fatigue [41]. Despite these challenges, some patients living in urban areas chose to manage their nutrition care independently. In contrast, some patients returning to rural and regional areas felt 'left behind' at the end of their treatment period. Studies have shown that cancer patients returning to rural areas can lack access to information and support [42]. Patients can struggle when moving away from their specialist cancer centre, highlighting the sense of security in being provided continuity of care [43]. Studies exploring psychosocial support needs of patients have identified a lack of information relevant to rural communities, with information and resources provided described as being urban-centric [44]. This emphasises the importance of understanding the unique informational needs of patients and carers, and ensuring they feel confident in being provided with continuity of care.

The longitudinal study design provided a greater understanding of patient and carer experiences as they changed throughout treatment and recovery. Interviews conducted two weeks after treatment completion meant that patients having surgery and PORT were not interviewed in between the two treatment modalities. While purposive sampling was used to gain a greater understanding of experiences between different treatment modalities, a limitation of this study is that greater focus was inadvertently placed on patient and carer experience throughout radiotherapy. To gain a greater understanding of the experiences of patients and carers of patients having surgery, future studies should explore experiences prior to the commencement of further treatment. The study included a greater male to female ratio (3:1) of patient participants, and greater female to male ratio (13:2) of carer participants. This reflects the gender distribution of the HNC patient and carer population but limited the opportunity to explore experiences of both roles from the perspective of the opposite gender.

As participants were followed up by the one interviewer, rapport was developed over time which may have resulted in more honest responses in interviews conducted at later time points [45]. To reduce individual bias, reflexive analysis based on Gadamerian hermeneutic inquiry was used to develop understanding of patient and carer experiences. This included recognition of the influences that the principal investigator brought to the study and the impact on results generated.

\section{Conclusion}


This study highlights tensions in the care relationships between patients, carers and healthcare professionals in the provision of nutrition care, as well as the changing nutritional needs and focus of patients throughout their treatment and recovery journey. There is a need to co-design strategies to improve nutrition care delivery for patients and carers both during and after treatment for HNC.

Strategies should consider practical and emotional support, offer peer support, and be easily accessible outside the hospital environment and inclusive of patients and carers regardless of geographical location, stage of treatment or recovery journey and individual health beliefs.

\section{Declarations}

\section{Acknowledgements}

We would like to thank all patient and carer participants for their valuable contribution to this project. We would also like to thank members of the multidisciplinary HNC clinic at the RBWH for supporting this project.

Funding: Principal investigator $(\mathrm{JH})$ was supported by a RBWH Postgraduate Research Scholarship from the RBWH Foundation for this project. The RBWH Foundation had no involvement in this study.

Conflicts of interest: The authors have no conflict of interest to disclose.

Availability of data and material: Not applicable

Code availability: Not applicable

\section{Author contributions:}

Conceptualisation: Joanne Hiatt, Teresa Brown, Judith Bauer, and Merrilyn Banks. Methodology: Joanne Hiatt and Judith Bauer. Data collection: Joanne Hiatt. Data analysis: Joanne Hiatt and Adrienne Young. Writing - original draft preparation: Joanne Hiatt

Writing - review and editing: Joanne Hiatt, Teresa Browns, Adrienne Young, Judith Bauer, and Merrilyn Banks. Funding acquisition: Joanne Hiatt. Resources: Joanne Hiatt. Supervision: Teresa Brown, Adrienne Young, Merrilyn Banks and Judith Bauer

Compliance with ethical standards: Ethics approval was obtained by the Royal Brisbane and Women's Hospital Research \& Ethics Committee, Brisbane and the Ethics Committee of the University of Queensland, Brisbane, Australia (HREC/17/QRBW/681), with Site Specific Approval for the study to be conducted at the Royal Brisbane and Women's Hospital.

Consent to participate and publish: Written informed consent forms were obtained from patient and carer participants prior to initiating research activities. 


\section{References}

1. Aupérin, A., Epidemiology of head and neck cancers: an update. Curr Opin Oncol, 2020. 32(3): p. 178186.

2. Hashibe, M., et al., Interaction between tobacco and alcohol use and the risk of head and neck cancer: pooled analysis in the International Head and Neck Cancer Epidemiology Consortium. Cancer Epidemiology and Prevention Biomarkers, 2009. 18(2): p. 541-550.

3. Young, D., et al., Increase in head and neck cancer in younger patients due to human papillomavirus (HPV). Oral oncology, 2015. 51(8): p. 727-730.

4. Marur, S. and A.A. Forastiere. Head and neck cancer: changing epidemiology, diagnosis, and treatment. in Mayo Clinic Proceedings. 2008. Elsevier.

5. Chasen, M.R. and R. Bhargava, A descriptive review of the factors contributing to nutritional compromise in patients with head and neck cancer. Supportive care in cancer, 2009. 17(11): p. 13451351.

6. Talwar, B., et al., Nutritional management in head and neck cancer: United Kingdom National Multidisciplinary Guidelines. The Journal of Laryngology \& Otology, 2016. 130(S2): p. S32-S40.

7. Bressan, V., et al., The life experience of nutrition impact symptoms during treatment for head and neck cancer patients: a systematic review and meta-synthesis. Supportive Care in Cancer, 2017. 25(5): p. 16991712.

8. Britton, B., et al., Heads up: a pilot trial of a psychological intervention to improve nutrition in head and neck cancer patients undergoing radiotherapy. European journal of cancer care, 2017. 26(4): p. e12502.

9. Brown, T., et al., Tube feeding during treatment for head and neck cancer-Adherence and patient reported barriers. Oral Oncology, 2017. 72: p. 140-149.

10. Ullgren, H., et al., How family caregivers of cancer patients manage symptoms at home: A systematic review. International journal of nursing studies, 2018. 85: p. 68-79.

11. Hiatt, J.S., et al., Patient and carer experience of nutrition care throughout treatment for head and neck cancer: a systematic qualitative review and thematic synthesis. Supportive Care in Cancer, 2020: p. 1-15.

12. Muylaert, C.J., et al., Narrative interviews: an important resource in qualitative research. Rev Esc Enferm USP, 2014. 48 Spec No. 2: p. 184-9.

13. Braun, V. and V. Clarke, Reflecting on reflexive thematic analysis. Qualitative Research in Sport, Exercise and Health, 2019. 11(4): p. 589-597. 
14. Braun, V. and V. Clarke, One size fits all? What counts as quality practice in (reflexive) thematic analysis? Qualitative research in psychology, 2020: p. 1-25.

15. Koch, T., Implementation of a hermeneutic inquiry in nursing: philosophy, rigour and representation. Journal of advanced nursing, 1996. 24(1): p. 174-184.

16. Brown, T.E., et al., Validated swallowing and nutrition guidelines for patients with head and neck cancer: identification of high-risk patients for proactive gastrostomy. 2013. 35(10): p. 1385-1391.

17. Findlay, M., J. Bauer, and T. Brown, Evidence-based practice guidelines for the nutritional management of adult patients with head and neck cancer-Cancer Guidelines Wiki. Wikicancerorgau. 2015. wiki. cancer. org. au/australia/COSA: Head_and_neck_cancer_nutrition_guidelines. Accessed, 2015. 20.

18. Booth, A., et al., COREQ (consolidated criteria for reporting qualitative studies). Guidelines for reporting health research: a user's manual, 2014: p. 214-26.

19. Lang, H., et al., The psychological experience of living with head and neck cancer: a systematic review and meta-synthesis. Psycho-oncology, 2013. 22(12): p. 2648-2663.

20. Islahudin, F., I.A. Shahdan, and S. Mohamad-Samuri, Association between belief and attitude toward preference of complementary alternative medicine use. Patient preference and adherence, 2017. 11: p. 913.

21. Keene, M.R., et al., Complementary and alternative medicine use in cancer: A systematic review. Complementary therapies in clinical practice, 2019. 35: p. 33-47.

22. Jones, E., et al., Exploring the use of complementary and alternative medicine in cancer patients. Integrative cancer therapies, 2019. 18: p. 1534735419846986.

23. Ryan, A.M., et al., Effects of weight loss and sarcopenia on response to chemotherapy, quality of life, and survival. Nutrition, 2019. 67: p. 110539.

24. Lazarus, R.S. and S. Folkman, Stress, appraisal, and coping. 1984: Springer publishing company.

25. Jackson, W., et al., Characterization of changes in total body composition for patients with head and neck cancer undergoing chemoradiotherapy using dual-energy x-ray absorptiometry. Head \& neck, 2014. 36(9): p. 1356-1362.

26. Lonkvist, C.K., et al., Progressive resistance training in head and neck cancer patients undergoing concomitant chemoradiotherapy. Laryngoscope investigative otolaryngology, 2017. 2(5): p. 295-306.

27. Boshier, P.R., et al., Assessment of body composition and sarcopenia in patients with esophageal cancer: a systematic review and meta-analysis. Diseases of the Esophagus, 2018. 31(8): p. doy047. 
28. Findlay, M., et al., "Completely and utterly flummoxed and out of my depth": patient and caregiver experiences during and after treatment for head and neck cancer-a qualitative evaluation of barriers and facilitators to best-practice nutrition care. Supportive Care in Cancer, 2020: p. 1-10.

29. Hazzard, E., et al., The patient experience of having a feeding tube during treatment for head and neck cancer: A systematic literature review. 2019. 33: p. 66-85.

30. Ang, S.Y., et al., Patients and home carers' experience and perceptions of different modalities of enteral feeding. Journal of clinical nursing, 2019. 28(17-18): p. 3149-3157.

31. White, S. and L. Brereton, Examining the role of patient values in decisions about long-term enteral feeding: A qualitative study. Clinical Nutrition, 2018. 37(3): p. 1046-1052.

32. Ang, S.Y., et al., Health care professionals' perceptions and experience of initiating different modalities for home enteral feeding. Clinical nutrition ESPEN, 2019. 30: p. 67-72.

33. Williams, G.F., et al., Patients' experience of enteral feeding following (chemo) radiotherapy for head and neck cancer: A qualitative study. Clinical Nutrition, 2019. 38(3): p. 1382-1389.

34. Egestad, H., The significance of fellow patients for head and neck cancer patients in the radiation treatment period. European Journal of Oncology Nursing, 2013. 17(5): p. 618-624.

35. Malloy-Weir, L.J., et al., Empirical relationships between health literacy and treatment decision making: a scoping review of the literature. Patient education and counseling, 2015. 98(3): p. 296-309.

36. Pritlove, C., et al., Cooking for Vitality: Pilot Study of an Innovative Culinary Nutrition Intervention for Cancer-Related Fatigue in Cancer Survivors. Nutrients, 2020. 12(9): p. 2760.

37. Sheridan, S.L., et al., Interventions for individuals with low health literacy: a systematic review. Journal of health communication, 2011. 16(sup3): p. 30-54.

38. Gao, R.W., J.D. Smith, and K.M. Malloy, Head and Neck Cancer and Social Media: The Patient Experience and Cancer Survivorship. The Laryngoscope, 2020.

39. Balfe, M., et al., Social networks, social support and social negativity: A qualitative study of head and neck cancer caregivers' experiences. European journal of cancer care, 2017. 26(6): p. e12619.

40. Baker, P., et al., 'Getting back to normal'or 'a new type of normal'? A qualitative study of patients' responses to the existential threat of cancer. European journal of cancer care, 2016. 25(1): p. 180-189.

41. O'Baugh, J., et al., 'Being positive': perceptions of patients with cancer and their nurses. Journal of Advanced Nursing, 2003. 44(3): p. 262-270.

42. Butow, P.N., et al., Psychosocial well-being and supportive care needs of cancer patients living in urban and rural/regional areas: a systematic review. Supportive Care in Cancer, 2012. 20(1): p. 1-22. 
43. Molassiotis, A. and M. Rogers, Symptom experience and regaining normality in the first year following a diagnosis of head and neck cancer: a qualitative longitudinal study. Palliative \& supportive care, 2012. 10(3): p. 197.

44. Gunn, K., et al., Psychosocial service use: a qualitative exploration from the perspective of rural Australian cancer patients. Supportive Care in Cancer, 2013. 21(9): p. 2547-2555.

45. Winiarska, A., Qualitative longitudinal research: Application, potentials and challenges in the context of migration research. 2017. 